\title{
Déficit de vitamina B12 en la práctica psiquiátrica
}

\author{
Cristian David Vargas-Upegui' ${ }^{1}$ Daniel Noreña-Rengifo²
}

\section{RESUMEN}

Es común la solicitud de los niveles de vitamina B12 en pacientes evaluados por psiquiatría y específicamente en las unidades de pacientes agudos, pero, a pesar de la creciente literatura disponible sobre la correlación entre esta hipovitaminosis y los síntomas neuropsiquiátricos, aún existen muchas dudas sobre los grupos poblacionales de riesgo que se deben tamizar, el tipo de examen que se debe solicitar, la forma de hacer el tratamiento y la utilidad real de la reposición vitamínica en la resolución de los síntomas afectivos, cognitivos o psicóticos. Este artículo es una revisión narrativa de la literatura disponible con el objetivo de poder evaluar de forma crítica los interrogantes teóricos y prácticos más relevantes para el psiquiatra actual, con énfasis en la evidencia sobre la correlación entre niveles bajos de vitamina B12 y déficit cognitivo, depresión y alogunos trastornos psicóticos.

\section{PALABRAS CLAVE}

Cianocobalamina; Demencia; Depresión; Hipovitaminosis; Vitamina B12

\section{SUMMARY}

\section{Vitamin B12 deficiency in psychiatric practice}

Levels of vitamin B12 are commonly requested in psychiatric patients, specifically in units for acute patients. Despite the abundant literature available about the correlation between this hypovitaminosis and neuro-psychiatric symptoms, there are still many doubts about the risk

\footnotetext{
1 Psiquiatra, Universidad de Antioquia. Candidato a MSc en Terapias de tercera generación, Universidad Internacional de Valencia. España. Docente de la Universidad de Antioquia y la Universidad Pontificia Bolivariana. Grupo de Investigación en Psiquiatría (GIPSI), Medellín, Colombia.

2 Residente Radiología, Universidad de Antioquia, Medellín, Colombia.

Correspondencia: Cristian David Vargas-Upegui; vargasupegui@gmail.com
}

Recibido: 23 de agosto de 2016

Aceptado: agosto 23 de 2016

Cómo citar: Vargas-Upegui CD, Noreña-Rengifo BD. Déficit de vitamina B12 en la práctica psiquiátrica. latreia. 2017 0ct-Dic;30(4):391-403. DOI 10.17533/udea.iatreia.v30n4a04. 
groups that should be screened, the tests that should be applied, how to treat, and the real usefulness of $\nabla$ itamin supplementation in the resolution of affective, cognitive or psychotic symptoms. This article presents a narrative review of the available literature, and summarizes the main recommendations about theoretical and practical questions concerning this subject matter, with emphasis on the correlation between low levels of vitamin B12 and cognitive impairment, depression and psychotic disorders.

\section{KEY WORDS}

Cyanocobalamin; Dementia; Depression; Vitamin B12; Vitamin Deficiency

\section{RESUMO}

\section{Déficit de Vitamina B12 na prática psiquiátrica}

É comum a solicitação de níveis de vitamina B12 em pacientes avaliados por psiquiatria e especificamente nas unidades de pacientes agudos, mas apesar da crescente literatura disponível sobre a correlação entre esta hipovitaminose e os sintomas neuropsiquiátricos, ainda existem muitas dúvidas sobre os grupos populacionais de risco que devem tamisar, o tipo de exame que se deve solicitar, a forma de fazer o tratamento e a real utilidade da reposição vitamínica na resolução dos sintomas afetivos, cognitivos ou psicóticos. Este artigo pretende fazer uma revisão narrativa da literatura disponível com o objetivo de poder avaliar de forma crítica os interrogantes teóricos e práticos mais relevantes para o psiquiatra atual, fazendo ênfase na evidência sobre a correlação entre níveis baixos de vitamina B12 e déficit cognitivo, depressão e alguns transtornos psicóticos.

\section{PALAVRAS CHAVE}

Cianocobalamina; Demência; Depressão; Hipovitaminose; Vitamina B12

\section{INTRODUCCIÓN}

La historia de la relación entre hipovitaminosis y síntomas neuropsiquiátricos no es nueva; la discusión ha cambiado desde el descubrimiento de las vitaminas en 1940 hasta la fecha, miogrando desde las hipótesis que las vinculan fuertemente con los síntomas psiquiátricos hasta las teorías más antivitamínicas; por ejemplo, en los años 70 se llegó a hablar de la terapia ortomolecular y megavitamínica como curativa de la esquizofrenia (1). Es común en la práctica psiquiátrica la solicitud de los niveles de vitamina B12, pero no existe consenso frente a las siguientes preguntas: ¿cuándo solicitar dicha medición?, ¿a quién tamizar?, ¿cómo interpretar los resultados?, ¿a quién hacerle reposición?, ¿cómo proceder cuando el paciente no es candidato a un tratamiento por inyección? y ¿cuáles son los síntomas de la hipovitaminosis B12 en neuropsiquiatría? Este artículo presenta una revisión narrativa de la literatura disponible con el objetivo de poder evaluar de forma crítica los interrogantes planteados.

\section{MATERIALES Y MÉTODOS}

Se hizo una revisión narrativa en las principales bases de datos (Pubmed, EMBASE y LILACS) de los artícuIos originales, revisiones y metaanálisis acerca de la relación entre el déficit de vitamina B12 y los síntomas neuropsiquiátricos. Se utilizaron los siguientes términos MESH: B12 vitamin, cyanocobalamin, neuropsychiatric symptoms, dementia, depression. Dos investigadores hicieron la selección del material; se complementó la revisión con textos básicos y referencias de los principales autores.

\section{NEUROBIOLOGÍA DEL DÉFICIT DE VITAMINA B12}

La vitamina B12 o cobalamina es una vitamina hidrosoluble producida por bacterias anaerobias; se encuentra en las proteínas animales y su reserva total en los humanos es de 2-5 mg (1 mg en el hígado). Su absorción en el enterocito del íleon distal depende del factor intrínseco, y la prevalencia mundial del déficit es variable: desde 7,8 \% en Israel hasta $49 \%$ en India. Esta carencia nutricional es mucho más común en niños, ancianos $(2,3)$ y mujeres embarazadas (4). En un estudio llevado a cabo por el Departamento de Nutrición de la Escuela de Salud Pública de Harvard en la población escolar colombiana, se encontró que hay una alta asociación entre niveles bajos de vitamina B12 y el estrato socioeconómico, lo cual sugiere 
que el diagnóstico de esta hipovitaminosis puede ser más frecuente en las poblaciones que tienen alta inseguridad alimentaria (5). T radicionalmente se ha sabido que la causa más frecuente del déficit es la mala absorción, pero posiblemente en nuestro medio la carencia nutricional sea un factor más relevante (5). Es importante tener en cuenta que la identificación de síntomas neurológicos (parestesias, debilidad muscular $y$ ataxia) y los hallazgos de laboratorio (aumento del volumen corpuscular medio, macroovalocitos $y$ neutrófilos hipersegmentados) pueden ser manifestaciones muy tardías de la disminución de los niveles de vitamina B12 (6); por ello, se plantea la tamización en grupos con factores de riesgo como la estrategia más utilizada y costo-efectiva para detectar la hipovitaminosis (7).

La cobalamina tiene un papel importante en diferentes vías metabólicas: es necesaria en la transformación del metil-tetrahidrofolato a tetrahidrofolato, que es la forma activa del ácido fólico; por ello, los niveles bajos de vitamina B12 generan también una depleción funcional del ácido fólico disponible, fenómeno conocido como "trampa de los folatos” (8) (figura 1).

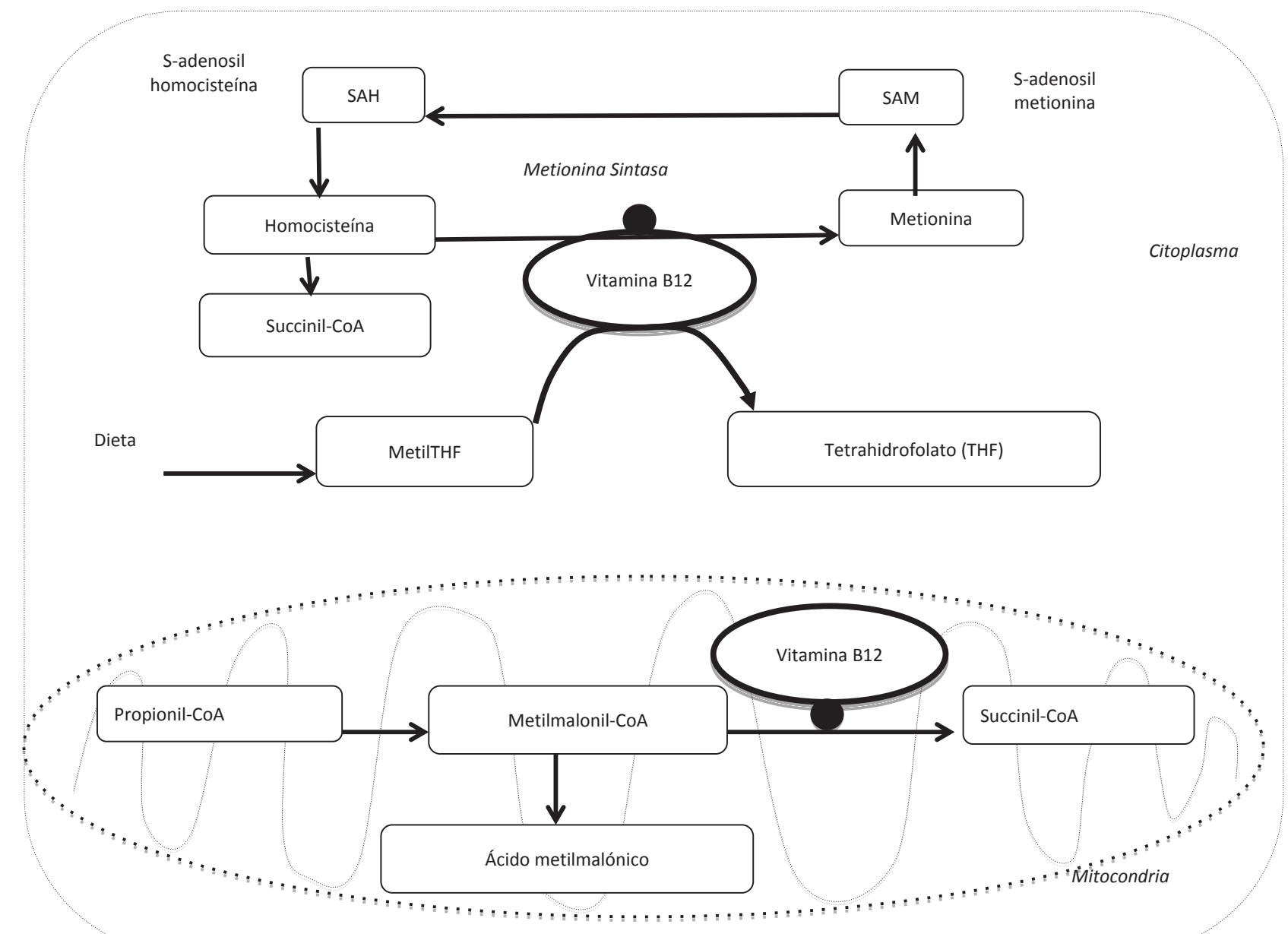

Figura 1. Principales vías metabólicas de la vitamina B12 
Ambas vitaminas promueven la síntesis de tetrahidrobiopterina, que a su vez participa como cofactor en la vía de la tirosina y el triptófano, necesarios para la producción de los neurotransmisores norepinefrina y serotonina. EI folato facilita la generación de S-adenosil metionina (SAM), que es un donador de grupos metilo necesarios para la formación de los neurotransmisores mencionados previamente; además, promueve la síntesis del ADN y de los fosfolípidos de membrana, receptores, canales y segundos mensajeros. Estas alteraciones metabólicas se reflejan en lesiones de la mielina, disminución del factor de crecimiento mielotrófico, lesión endotelial, apoptosis celular, disminución del GABA, disfunción de la barrera hematoencefálica y metilación de fosfolípidos en las membranas de las neuronas $(4,9)$.

Cuando se evalúa con neuroimágenes a los pacientes con déficit de vitamina B12 se observan hiperintensidades periventriculares y subcorticales (leucoaraiosis), atrofia cerebral e infartos silenciosos $(4,10,11)$. Un estudio de 1019 pacientes sin demencia mostró una correlación inversamente proporcional entre los niveles de vitamina B12 y las lesiones periventriculares y subcorticales en la sustancia blanca, incluso después de haber controlado los factores cardiovasculares de confusión (3). En un estudio de 271 individuos mayores de 70 años y con deterioro cognitivo leve, se encontró que la suplencia de vitamina B12, vitamina B6 y ácido fólico durante 24 meses disminuyó la progresión de la atrofia cerebral en comparación con el grupo que recibió placebo (12).

\section{SÍNTOMAS NEUROPSIQUIÁTRICOS ASOCIADOS AL DÉFICIT DE VITAMINA B12}

\section{Déficit cognitivo leve y síndrome demencial}

El déficit de vitamina B12 no solo se asocia con la afectación de las vías de producción de neurotransmisores y la estabilidad de la membrana neuronal, sino también con lesiones cerebrales identificadas por neuroimágenes, lo que puede relacionarse con un compromiso cognitivo $(11,13-17)$. Una revisión sistemática en 2012 que incluyó 43 estudios halló que niveles inferiores a $150 \mathrm{pg} / \mathrm{mL}$ se asociaron con déficit cognitivo (9), en tanto que los menores de $250 \mathrm{pg} / \mathrm{mL}$ se han asociado con demencia tipo Alzheimer (18), demencia vascular y enfermedad de Parkinson (19). Por otra parte, algunos estudios han mostrado que la demencia aumenta el riesogo de tener déficit vitamínico y que cuanto más grave sea el compromiso en el test mini-mental más bajos son los niveles séricos de vitamina B12 (20). Otros autores también han asociado el déficit con demencia frontotemporal (21).

En literatura reciente se propone que la deficiencia de vitamina B12 no diagnosticada puede ser una oportunidad perdida para la prevención de la demencia y de accidentes cerebrovasculares (22). Existe una relación causal entre hipovitaminosis y déficit cognitivo (23), pero se ha propuesto que esto, más que una causa, es una comorbilidad por las condiciones de vida, la alimentación y el cuidado de los pacientes con demencia (24). Aunque alounos estudios no encontraron asociación entre el déficit de vitamina B12 y el riesgo de demencia, por ejemplo, de tipo Alzheimer, en un estudio en 2007 con una cohorte de 2741 individuos mayores de 65 años se halló que tener niveles bajos de vitamina B12 se asociaba con deterioro coonitivo más rápido y que la duplicación de los niveles de ácido metilmalónico esturo asociada con una disminución cognitiva más rápida en más del 50 \% de los casos. Este hallazơo continuó siendo sionnificativo después de ajustar por todos los marcadores vitamínicos simultáneos (25).

La evidencia muestra que es común la comorbilidad entre déficit coognitivo e hipovitaminosis B12 (26) y que la presencia de la misma en estudios longitudinales se asocia con la velocidad del deterioro (27), pero al evaluar qué tan efectivo es el tratamiento de reposición para revertir el daño, los resultados no son tan promisorios (9). En la tablas 1 y 2 se pueden observar algunos de los principales estudios prospectivos y de los controlados con placebo que han evaluado el efecto de la reposición vitamínica en el déficit cognitivo; no se incluyeron estudios retrospectivos debido a la falta de datos y a las limitaciones metodológicas. Como se puede observar, a pesar de que los diseños de los estudios tienen dificultades, por ser heterogéneos y utilizar diferentes dosis de reposición y tiempos, ningún estudio muestra que el tratamiento de reposición en pacientes con demencia y déficit cognitivo leve revierta el daño cognitivo, por lo que son necesarios estudios de mejor calidad (28-32). Algunos autores han planteado la posibilidad de que el efecto 
de la reposición de vitamina B12 dependa del estadio clínico de la demencia y que exista una "ventana terapéutica” para revertir el daño (33). Dos estudios mostraron que la reposición de esta vitamina era más efectiva cuando el déficit cognitivo era inferior a un año, con normalización de síntomas según la Dementia Rating Scale (33) y con ganancia de hasta 6 puntos en el test mini-mental (34).

No se dispone de evidencia sobre la utilización de vitamina B12 como medida preventiva del daño cognitivo en la población general ni sobre cambios en la función cognitiva en personas asintomáticas sin déficit o con niveles bajos. Además, en pacientes hospitalizados con hiporitaminosis B12, no se ha logrado establecer una relación clara con demencia, tal y como se demostró en una investigación del 2015 hecha en 666 pacientes (35).

Un estudio llevado a cabo en 2008 con 36 pacientes remitidos con demencia leve a moderada y comorbilidad con hipovitaminosis B12 demostró mejoría de los potenciales auditivos evocados y del deterioro cognitivo posterior a la reposición de vitamina B12 (11).

Las inconsistencias entre los estudios observacionales y los de intervención con vitamina B12 en pacientes con déficit cognitivo se pueden explicar por varias

Tabla 1. Estudios que evalúan el efecto de la reposición de vitamina B12 en los síntomas cognitivos

\begin{tabular}{|c|c|c|c|c|c|}
\hline Estudio & Diseño & $\begin{array}{l}\text { pacientes con } \\
\text { demencia }(\mathbf{n})\end{array}$ & $\begin{array}{l}\text { Métodos de } \\
\text { medición }\end{array}$ & $\begin{array}{c}\text { Porcentaje de } \\
\text { déficit de vitamina } \\
\text { B12 }\end{array}$ & $\begin{array}{l}\text { Respuesta clínica a la } \\
\text { reposición }\end{array}$ \\
\hline $\begin{array}{l}\text { Cunha y colaboradores } \\
1990 \text { (38) }\end{array}$ & Prospectivo & 110 & $\begin{array}{l}\text { Niveles séricos y } \\
\text { MMES }\end{array}$ & 16 & No mejoría \\
\hline $\begin{array}{l}\text { Cunha y colaboradores } \\
1995 \text { (39) }\end{array}$ & Prospectivo & 181 & $\begin{array}{l}\text { Niveles séricos y } \\
\text { MMES }\end{array}$ & $\begin{array}{c}25 \\
(<200 \mathrm{pg} / \mathrm{mL})\end{array}$ & $\begin{array}{c}\text { Mejoría en } 9 / 13 \text { pacientes } \\
\text { (con déficit leve y menos } \\
\text { de } 2 \text { años) }\end{array}$ \\
\hline $\begin{array}{l}\text { Teunisse y colaborado- } \\
\text { res } 1996(40)\end{array}$ & Prospectivo & 170 & $\begin{array}{l}\text { Niveles séricos, } \\
\text { otras medidas y } \\
\text { MMES }\end{array}$ & 15 & No mejoría \\
\hline $\begin{array}{l}\text { Kalita y colaboradores } \\
2008 \text { (11) }\end{array}$ & Prospectivo & 36 & $\begin{array}{l}\text { Niveles séricos, } \\
\text { otras medidas y } \\
\text { MMES }\end{array}$ & $88^{*}(<211 \mathrm{pg} / \mathrm{mL})$ & $\begin{array}{c}\text { Mejoría en todos los } \\
\text { pacientes }\end{array}$ \\
\hline
\end{tabular}

MMES: Minimental Examination Scale; *Se hizo medición sérica a 34 pacientes de la muestra total

Tabla 2. Estudios clínicos controlados con placebo que evalúan el efecto de la reposición de vitamina B12 en los síntomas cognitivos

\begin{tabular}{|c|c|c|c|c|c|}
\hline Estudios y población & $\begin{array}{l}\text { Dosis de reposición } \\
\text { B12 }\end{array}$ & Tratamiento (n) & Placebo (n) & $\begin{array}{l}\text { Métodos de } \\
\text { medición }\end{array}$ & $\begin{array}{l}\text { Respuesta clínica a la } \\
\text { reposición }\end{array}$ \\
\hline $\begin{array}{l}\text { Seal y colaboradores } \\
2002 . \\
\text { Trastornos de ma- } \\
\text { labsorción, enferme- } \\
\text { dades neurológicas } \\
\text { progresivas o enfer- } \\
\text { medades terminales } \\
\text { (28) }\end{array}$ & $\begin{array}{l}\text { 10-50 } \mu \mathrm{g} / \text { día por } 1 \\
\text { mes }\end{array}$ & 20 & 11 & MMES & $\begin{array}{l}\text { No hubo diferencias ni } \\
\text { cambios en los niveles } \\
\text { de } \mathrm{Hc}\end{array}$ \\
\hline $\begin{array}{l}\text { Lehmann y colabora- } \\
\text { dores } 2003 \text {. } \\
\text { Déficit cognitivo leve } \\
\text { (29) }\end{array}$ & 2 mg oral por 270 días & 30 & 35 & MMES & No hubo diferencia clínica \\
\hline
\end{tabular}


Tabla 2. Estudios clínicos controlados con placebo que evalúan el efecto de la reposición de vitamina B12 en los síntomas cognitivos (Continuación)

\begin{tabular}{|c|c|c|c|c|c|}
\hline $\begin{array}{l}\text { Dangour y cola- } \\
\text { boradores } 2015 . \\
\text { Individuos de } 75 \text { o } \\
\text { más años con déficit } \\
\text { moderado de vitami- } \\
\text { na B12 (41) }\end{array}$ & $1 \mathrm{mg}$ por 12 meses & 99 & 102 & CVLT & $\begin{array}{l}\text { No hubo diferencia esta- } \\
\text { dísticamente significativa }\end{array}$ \\
\hline $\begin{array}{l}\text { Van Uffelen y colabo- } \\
\text { radores } 2008 . \\
\text { Déficit cognitivo leve } \\
\text { (30) }\end{array}$ & $0,4 \mathrm{mg}$ oral por 1 año & 78 & 74 & $\begin{array}{l}\text { MMES y otras } \\
\text { pruebas }\end{array}$ & $\begin{array}{l}\text { No hubo diferencias en el } \\
\text { MMES. } \\
\text { Mejoría en la DSST en } \\
\text { mujeres. }\end{array}$ \\
\hline
\end{tabular}

\begin{tabular}{|c|c|c|c|c|c|}
\hline $\begin{array}{l}\text { Aisen y colaboradores } \\
\text { 2008. Alzheimer (31) }\end{array}$ & $1 \mathrm{mg}$ por 18 meses & 202 & 138 & ADAS test & $\begin{array}{l}\text { No hubo diferencia clínica } \\
\text { ni en los niveles de Hc. }\end{array}$ \\
\hline $\begin{array}{l}\text { Brady y colaboradores } \\
2009 \text {. Deterioro cog- } \\
\text { nitivo e insuficiencia } \\
\text { renal crónica. (32) }\end{array}$ & $2 \mathrm{mg}$ por 5 años & 339 & 320 & $\begin{array}{l}\text { Entrevista } \\
\text { telefónica } \\
\text { cognitiva }\end{array}$ & $\begin{array}{l}\text { No hubo diferencia clínica } \\
\text { ni en los niveles de Hc. }\end{array}$ \\
\hline $\begin{array}{l}\text { Van Dyck y colabora- } \\
\text { dores 2009. Demencia } \\
\text { (27) }\end{array}$ & $\begin{array}{l}1000 \mu \mathrm{g} / \mathrm{IM} / \text { día por } 1 \\
\text { semana, luego sema- } \\
\text { nal por } 16 \text { semanas }\end{array}$ & 28 & 28 & $\begin{array}{l}\text { DRS } \\
\text { BPRS } \\
\text { GDS }\end{array}$ & $\begin{array}{l}\text { No hubo diferencias sig- } \\
\text { nificativas. Cambios en las } \\
\text { patrones hematológicos }\end{array}$ \\
\hline
\end{tabular}

DRS: Dementia Rating Scale. BPRS: Brief Psychiatry Rating Scale. GDS: Geriatric Depression Scale. CVLT: California Verbal Learning Test. ADAS: Alzheimer Disease Assessment Scale. DSST: Velocidad del procesamiento de información, por test de sustitución de símbolos digitales, Hc: homocisteía

razones, como el bajo poder estadístico de los estudios, la reposición con dosis insuficientes, los tratamientos cortos (menos de 6 meses), los diferentes tipos de escalas de medición del efecto y el mal control de los factores de confusión (36).

En conclusión, hasta la fecha ninguna evidencia muestra una relación causal entre la hipovitaminosis B12 y el déficit cognitivo, pero existe evidencia de la comorbilidad del déficit de vitamina B12 en pacientes con demencia y déficit cognitivo leve, por lo que sería un grupo de riesgo que siempre se debería tamizar. Se necesita nueva evidencia que determine si la reposición vitamínica puede revertir el daño cognitivo (37).

\section{DEPRESIÓN}

Se ha asociado la hipovitaminosis de B12 con la depresión (42-44). En el estudio de Rotterdam se encontró que el déficit de vitamina B12 es un factor de riesgo independiente para depresión (45). Otros autores han planteado que los niveles bajos predicen el inicio del episodio afectivo; por tanto, sugieren que el déficit de esta vitamina puede ser un factor de riesgo independiente para depresión en pacientes mayores de 65 años $(46,47)$. Estudios en pacientes ancianos muestran que tener niveles séricos de vitamina B12 inferiores a 180 $\mathrm{pg} / \mathrm{mL}$ aumenta entre 2 y 2,5 veces el riesgo de depresión, independientemente de los niveles de folato y homocisteína (48).

A pesar de que algunas investigaciones han mostrado que no hay mayor efecto sobre los síntomas depresivos con el tratamiento de reposición (vitamina B12, B6 y folato), un estudio longitudinal de 7 años que incluyó 3503 pacientes ancianos mostró que cada 10 miliogramos adicionales de vitamina B6 y 10 microgramos de vitamina B12 en la ingesta se asociaron con $2 \%$ menos de probabilidad de síntomas depresivos por año de seguimiento (49). Las investigaciones que tratan de resolver esta pregunta sobre el efecto de la reposición en la mejoría de los síntomas depresivos son muy heterogéneas $\mathrm{y}$ tienen dosis tan bajas como de 15 $\mu \mathrm{g} / \mathrm{día}$, las cuales posiblemente no tienen un efecto clínicamente significativo (50). Un estudio en pacientes mayores de 65 años, que recibieron vitaminas B12, B6 
y ácido fólico versus placebo como medida preventiva para la depresión, no mostró cambios significativos en la incidencia ni en la gravedad de los síntomas (51). En un estudio hecho en un suborrupo especial, como es la población infectada por $\mathrm{VIH}$, no se encontró mejoría en la escala de depresión de Hamilton después de la suplementación de vitamina B12 en pacientes con deficiencia, pero se halló que los pacientes que tenían comorbilidad con tuberculosis presentaban un riesgo más alto de hipovitaminosis B12, lo cual sugiere que este grupo puede ser otra población de alto riesogo (52).

En un estudio reciente que evaluó 391 ancianos de 64 a 91 años se encontró que había una asociación entre el afecto positivo y los niveles altos de vitamina B12 y áciđo fólico, específicamente en población anciana ambulatoria (53).

En conclusión, aunque hay asociación entre hipovitaminosis B12 y depresión mayor en pacientes ancianos, aún no es claro el papel terapéutico de la reposición vitamínica en la mejoría clínica de la depresión, por lo que podría ser considerado como un factor precipitante $y$ no como el factor causal único; sin embargo, se requieren más estudios para confirmar estos hallazgos (54).

\section{Psicosis, delirio y otros trastornos psiquiátricos}

En la literatura hay estudios que asocian los niveles séricos de vitamina B12 y psicosis; van desde reportes de casos hasta estudios observacionales (55). Las descripciones se han hecho principalmente en pacientes ancianos, y la psicopatología ha sido muy variada, incluyendo suspicacia, delirios místicos/religiosos, alucinaciones auditivas y visuales, trastornos formales del pensamiento $y$ tangencialidad (56), por lo que no hay un cuadro específico que haga sospechar el déficit vitamínico. La relación de psicosis secundaria con hipovitaminosis B12 no es tan fuerte según la evidencia actual (1).

La asociación con delirio es más antigua, ya que desde 1956 había descripciones de mejoría de este en pacientes con cuadros de anemia perniciosa, $y$ hay informes de casos en pacientes con y sin demencia sobreagregada al delirio (57). Un estudio prospectivo en pacientes con delirio que además tenían déficit de vitamina B12 identificó una mejoría en el minimental en más del 50 $\%$ de los casos y además se observaron cambios en el flujo cerebral después del suplemento vitamínico (58).
En un estudio efectuado en pacientes ambulatorios mayores de 60 años y con diagnóstico de demencia no se encontró mejoría con el suplemento vitamínico administrado durante 40 semanas, pero sí se demostró una disminución del riesgo de presentar delirio a las 6 semanas, según la Delirium Rating Scale (DRS); sin embargo, se requieren mayores estudios para determinar el papel de la hipovitaminosis B12 en la prevención y tratamiento del delirio en este grupo de pacientes (59).

Existen pocos informes de casos que asocien episodios maníacos con déficit de vitamina B12 y hay una hipótesis que plantea que la asociación es posible por la presencia de lesiones en la sustancia blanca descritas en los estudios de neuroimágenes (60-62). Falta evidencia que pueda apoyar esta hipótesis, pero algunos autores recomiendan hacer tamizaje en el primer episodio de manía, y con mayor indicación si no hay historia familiar de trastornos afectivos $(63,64)$. Se han informado en algunos estudios niveles séricos bajos de vitamina B12, pero en otros no se ha encontrado diferencia o han sido altos (60-62). Un estuđio reciente mostró que los niveles séricos y cerebrales de cobalamina y metilcobalamina estaban disminuidos en estos pacientes y que esto podría asociarse a la fisiopatología de esta enfermedad (65).

Hay pocos estudios que relacionen el déficit de vitamina B12 con otros trastornos psiquiátricos como el obsesivo compulsivo (66) o la depresión psicótica (67). En los reportes de casos de estas dos enfermedades los niveles séricos fueron inferiores a $200 \mathrm{pg} / \mathrm{mL}$. Alogunos artículos han descrito la aparición de catatonía como una manifestación neuropsiquiátrica en pacientes con déficit de vitamina B12, con niveles tan bajos como $1,07 \mathrm{pg} / \mathrm{mL}$ y tan altos como $150 \mathrm{pg} / \mathrm{mL}$ $(68,69)$, pero la evidencia está circunscrita solamente a informes de casos (64).

\section{TAMIZAJE Y NIVELES SÉRICOS}

La solicitud de los niveles de vitamina B12 es común en la práctica clínica de la psiquiatría y, según la evidencia actual, se recomienda hacer tamizaje en poblaciones de riesgo y no de forma rutinaria en todos los pacientes psiquiátricos. La Asociación Americana 
de Psiquiatría en su guía práctica para la evaluación psiquiátrica de adultos recomienda medir los niveles de vitamina B12 en los pacientes con diagnóstico de demencia (70). Aún existen muchas dudas sobre la asociación con psicosis y con otras enfermedades psiquiátricas, en las que la mayoría de la evidencia está basada en reportes de casos, pero en el tamizaje se debe plantear la medición de los niveles de vitamina B12 en los pacientes con el primer episodio psicótico (7).

Otros grupos de pacientes que se deben tamizar son los que tienen alteraciones en la absorción intestinal y en la producción del factor intrínseco, historia de cirugía bariátrica, deficiencia genética, ingesta inadecuada, infección por Helicobacter pylori (63) y uso prolongado de fármacos como antagonistas $\mathrm{H} 2$, inhibidores de la bomba de protones, anticonvulsivantes y metformina $(3,4)$

En el contexto del hospital psiquiátrico, alogunos estudios en pacientes ancianos muestran la utilidad diagnóstica de medir los niveles séricos de vitamina B12; otros no concuerdan con ello, y algunas revisiones concluyen que no es una medición indicada en todos los pacientes evaluados por el psiquiatra y que el mayor beneficio se observa en grupos de alto riesgo, como los mayores de 65 años (71), los que usan psicoactivos $y$ alcohol $y$ los que tienen delirio $(57,59)$. Según la literatura, cuando un paciente tiene síntomas neuropsiquiátricos en el servicio de urgencias, el mayor beneficio del tamizaje se observa en ancianos, personas que consumen psicoactivos, sin antecedentes psiquiátricos conocidos o con problemas médicos recurrentes (5). En 2015 se publicó un estudio que evaluó 259 pacientes mayores de 60 años con diferentes trastornos neuropsiquiátricos (enfermedad de Alzheimer, demencia con cuerpos de Lewy, demencia frontotemporal, esquizofrenia, enfermedad de Parkinson, síndrome de dependencia al alcohol, trastorno afectivo bipolar y enfermedad de Creutzfeldt-Jakob); se encontró que $16 \%$ tenían niveles bajos de vitamina B12 (definida para este estudio como menores de $220 \mathrm{pg} / \mathrm{mL}$ ) (16).

En la literatura se han informado tres formas principales para evaluar el déficit de vitamina B12: medir sus niveles séricos, los del ácido metilmalónico (> $0,4 \mu \mathrm{mol} / \mathrm{L})$ y los de la homocisteína (>13 $\mu \mathrm{mol} / \mathrm{L}$ ) $(72,73)$. La medición sérica por radioinmunoensayo tiene la desventaja de los falsos negativos por formas inactivas no identificadas y por unión a proteínas, $y$ con esta técnica la deficiencia en los tejidos se subestima en un $50 \%$, aproximadamente (74). El ácido metilmalónico se acumula cuando hay deficiencia de cobalamina por un bloqueo en el paso metabólico desde metilmalonil-CoA a succinil-CoA; su medición se considera más específica, pero se eleva en pacientes con depleción de volumen o insuficiencia renal (6) (figura 1). Finalmente, la medición de los niveles de homocisteína y ácido metilmalónico es más sensible que la sola medición de cobalamina sérica: $95 \%$ versus $69 \%$; además, pueden reflejar también la deficiencia de folato (68).

El diagnóstico de hipovitaminosis B12 por medición de los niveles séricos permite la siguiente clasificación: 1) "bajos" cuando son menores de 150-200 pg/ $\mathrm{mL}(75) ; 2)$ "normales" cuando están por encima de $350 \mathrm{pg} / \mathrm{mL}$; en este último caso es improbable que los síntomas neuropsiquiátricos se expliquen por hipovitaminosis $(9,35,63)$; 3) un rango llamado "normal bajo" entre $150-200 \mathrm{pg} / \mathrm{mL}$ y $350 \mathrm{pg} / \mathrm{mL}$, en el que la mayoría de los estudios recomiendan medir el ácido metilmalónico para poder definir si existe o no una hipovitaminosis real $(9,35,63,76)$. Ante las dificultades para medir dicho ácido, algunos autores recomiendan la reposición vitamínica cuando los niveles son inferiores a $350 \mathrm{pg} / \mathrm{mL}$ (2). Estudios posteriores deberán evaluar la relación costo-efectividad de la reposición según los niveles de vitamina B12 o utilizando el ácido metilmalónico cuando sea necesario porque existe una alta probabilidad de que actualmente se esté administrando tratamiento a muchos pacientes que no lo requieren (77) (figura 2).

\section{TRATAMIENTO Y REPOSICIÓN}

Se han informado muchos esquemas de reposición que oscilan entre 125 y $2000 \mu \mathrm{g} /$ día, pero la mayoría proponen que la dosis promedio sea de $1000 \mu \mathrm{g} /$ día $(4,27,78)$. La estrategia más utilizada es la reposición parenteral (79), pero según una revisión Cochrane de 2005 la reposición oral es tan efectiva como la intramuscular (77). En este protocolo se recomienda administrar 1-2 mg/día durante 90-120 días por vía oral, y se sugiere para pacientes con alguna contraindicación o en quienes rechazan la vía intramuscular; su dosis es mucho más alta porque la absorción es solo del $20 \%$ de la vitamina disponible en la luz intestinal. 
Actualmente se recomienda el esquema de reposición con $1000 \mu$ g intramusculares semanalmente durante 8 semanas y luego mensualmente hasta que el déficit se corrija o de forma indefinida si la causa primaria persiste (4). Aunque no se encontraron estudios que compararan los diferentes esquemas, otro protocolo de reposición muy utilizado y recomendado en la literatura es la aplicación intramuscular diaria de 1000 $\mu$ g por 7 días, luego semanal por 4 semanas y después cada 1-3 meses según la gravedad (75). No hay claridad sobre los esquemas de reposición en casos de déficit subclínico. Es importante tener en cuenta que en casos de deficiencia no nutricional y cuando no es posible resolver la causa (por ejemplo, cirugía gástrica, deficiencia de factor intrínseco, entre otras) el tratamiento es a término indefinido. En los pacientes con enfermedad renal, podría preferirse la administración oral de $1000 \mu \mathrm{g}$ de metilcobalamina diaria o cada 12 horas. Aunque no hay un consenso mundial sobre la reevaluación de los niveles de vitamina B12 para identificar la respuesta al tratamiento, algunos autores recomiendan medirlos a las 4-6 semanas, y hacer controles de seguimiento cada 6 a 12 meses después de haber alcanzado los niveles normales (4).

\section{Depresión, déficit cognitivo o primer episodio psicótico}

¿Es grupo de riesgo?

Metformina, vegetarianismo, cirugía bariátrica, anticonvulsivantes, >65 años, gastritis atrófica, cirugía intestinal, consumo de psicoactivos y alcohol, sospecha de déficit nutricional.

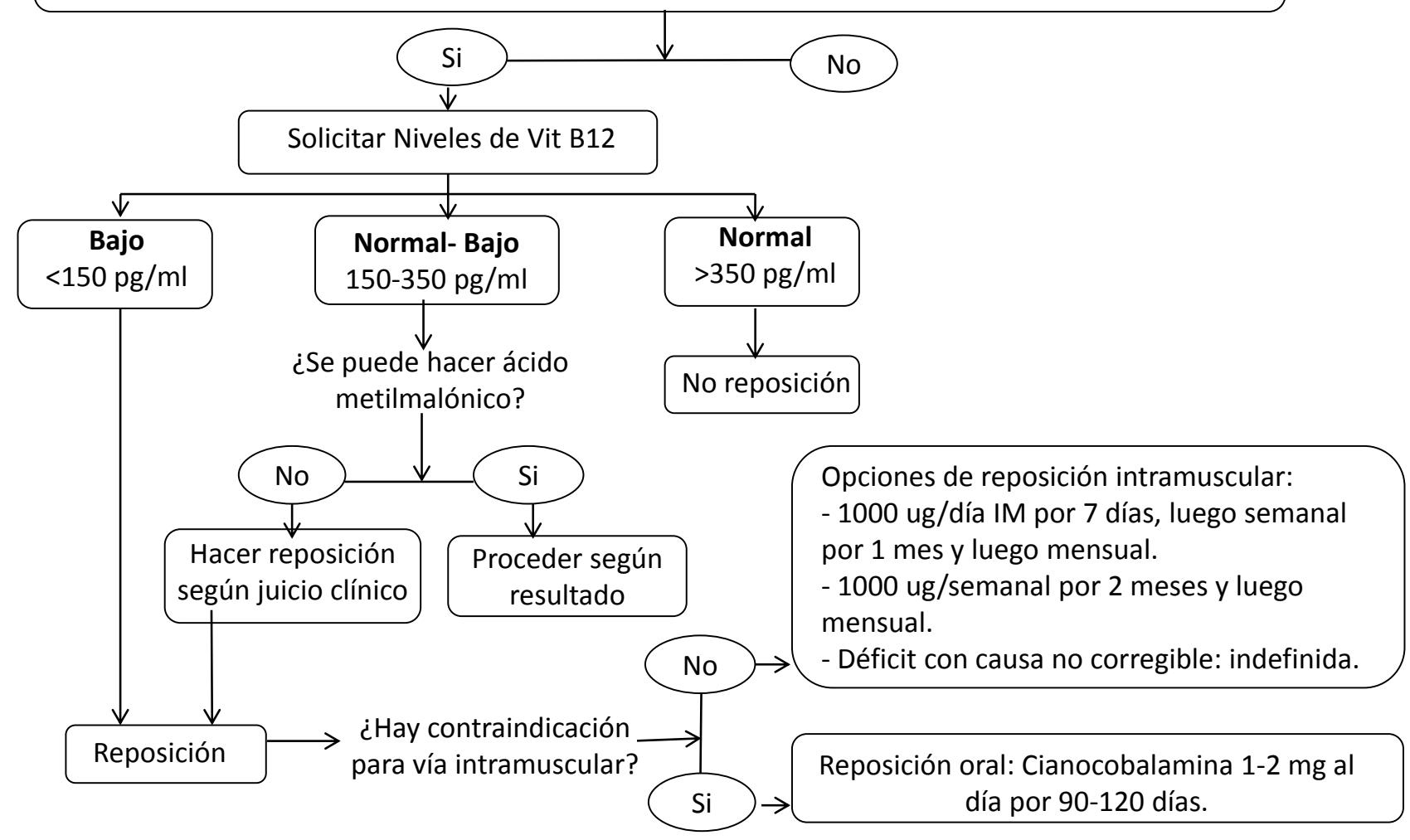

Figura 2. Propuesta de algoritmo de tamizaje y reposición de déficit de vitamina B12 en síntomas neuropsiquiátricos 


\section{CONCLUSIONES}

Existe asociación entre niveles bajos de vitamina B12 y demencia, pero hay poca evidencia sobre el efecto de la reposición vitamínica en los síntomas cognitivos, $y$ actualmente no hay pruebas suficientes para recomendar la administración de suplementos de esta vitamina como un tratamiento para la demencia o el deterioro cognitivo si no hay hipovitaminosis B12; sin embargo, algunos estudios muestran que corregir la hipovitaminosis B12 para mejorar el déficit cognitivo asociado a ella es más efectivo en los primeros 12 meses, $y$ que el tratamiento en pacientes con demencia disminuye el riesgo de delirio a las 6 semanas.

El déficit de vitamina B12 duplica el riesgo de depresión tardía en los ancianos, pero los estudios de reposición no muestran un efecto tan fuerte en la mejoría de los síntomas. La tamización midiendo los niveles de vitamina B12 se debe hacer según los factores de riesgo, $y$ se propone que la re posición sea intramuscular, aunque según las últimas revisiones también es posible hacerla por vía oral. De esta revisión se concluye que se requieren más estudios para aclarar muchas de las dudas actuales sobre el efecto de la reposición en la mejoría de la psicopatología y la funcionalidad de los pacientes.

\section{CONFLICTOS DE INTERESES}

Ninguno por declarar.

\section{REFERENCIAS BIBLIOGRÁFICAS}

1. Wang TY, Lee SY, Chen SL, Chang YH, Chen SH, Huang SY, et aI. Gender-specific association of the SLC6A4 and DRD2 gene variants in bipolar disorder. Int J Neuropsychopharmacol. 2014 Feb;17(2):211-22. DOI 10.1017/S1461145713001296.

2. Hin H, Clarke R, Sherliker P, Atoyebi W, Emmens K, Birks J, et al. Clinical relevance of low serum vitamin B12 concentrations in older people: the Banbury B12 study. Age Ageing. 2006 Jul;35(4):416-22.

3. Selhub J, Troen A, Rosenberg IH. B vitamins and the aging brain. Nutr Rev. 2010 Dec;68 Suppl 2:S112-8. DOI 10.1111/j.1753-4887.2010.00346.x.

4. Langan RC, Zawistoski KJ. Update on vitamin B12 deficiency. Am Fam Physician. 2011 Jun;83(12):1425-30.
5. Villamor E, Mora-Plazas M, Forero Y, Lopez-Arana S, Baylin A. Vitamin B-12 status is associated with socioeconomic level and adherence to an animal food dietary pattern in Colombian school children. J Nutr. $2008 \mathrm{JuI} ; 138(7): 1391-8$.

6. Reynolds E. Vitamin B12, folic acid, and the nervous system. Lancet Neurol. 2006 Nov;5(11):949-60.

7. LachnerC, Steinle NI, RegenoId WT. The neuropsychiatry of vitamin B12 deficiency in elderly patients. J Neuropsychiatry Clin Neurosci. 2012 Winter;24(1):5-15. DOI 10.1176/appi.neuropsych.11020052.

8. Crellin R, Bottiglieri T, Reynolds EH. Folates and psychiatric disorders. Clinical potential. Drugss. 1993 May;45(5):623-36.

9. Moore E, Mander A, Ames D, Carne R, Sanders K, Watters D. Cognitive impairment and vitamin B12: a review. Int Psychogeriatr. 2012 Apr;24(4):541-56. DOI 10.1017/S1041610211002511.

10. Johnson C, Droon T, McMahon FJ, UhI GR. Convergent genome wide association results for bipolar disorder and substance dependence. Am J Med Genet B Neuropsychiatr Genet. 2009 Mar;150B(2):182-90. DOI 10.1002/ajmg.b.30900.

11. Kalita J, Misra UK. Vitamin B12 deficiency neurological syndromes: correlation of clinical, MRI and cognitive evoked potential. J Neurol. 2008 Mar;255(3):3539. DOI 10.1007/s00415-008-0660-X.

12. Smith AD, Smith SM, de Jager CA, Whitbread P, Johnston C, Agacinski G, et al. Homocysteine-lowering by $\mathrm{B}$ vitamins slows the rate of accelerated brain atrophy in mild cognitive impairment: a randomized controlled trial. PLoS One. 2010 Sep;5(9):e12244. DOI 10.1371/journal.pone.0012244.

13. Osimani A, Berger A, Friedman J, Porat-Katz BS, Abarbanel JM. Neuropsychology of vitamin B12 deficiency in elderly dementia patients and control subjects. J Geriatr Psychiatry Neurol. 2005 Mar; 18(1):33-8.

14. Ravaglia G, Forti P, Maioli F, Martelli M, Servadei L, Brunetti N, et al. Homocysteine and folate as risk factors for dementia and Alzheimer disease. Am J Clin Nutr. 2005 Sep;82(3):636-43.

15. Levitt AJ, Karlinsky H. Folate, vitamin B12 and cognitive impairment in patients with Alzheimer's disease. Acta Psychiatr Scand. 1992 Oct;86(4):301-5.

16. Refsum H, Smith AD. Low vitamin B-12 status in confirmed Alzheimer's disease as revealed by serum 
holotranscobalamin. J Neurol Neurosurg Psychiatry. $2003 \mathrm{Jul} ; 74(7): 959-61$.

17. de Lau LM, Smith AD, Refsum H, Johnston C, Breteler MM. Plasma vitamin B12 status and cerebral whitematter lesions. J Neurol Neurosurg Psychiatry. 2009 Feb;80(2):149-57. DOI 10.1136/jnnp.2008.149286.

18. Seshadri S, Beiser A, Selhub J, Jacques PF, Rosenberg IH, D'Agostino RB, et al. Plasma homocysteine as a risk factor for dementia and Alzheimer's disease. $\mathrm{N}$ Engl J Med. 2002 Feb;346(7):476-83.

19. Whyte EM, Mulsant BH, Butters MA, Qayyum M, Towers A, Sweet RA, et al. Cognitive and behavioral correlates of low vitamin B12 levels in elderly patients with progressive dementia. Am J Geriatr Psychiatry. 2002 May-Jun;10(3):321-7.

20. Rigogs KM, Spiro A, Tucke K, Rush D. Relations of vitamin B-12, vitamin B-6, folate, and homocysteine to cognitive performance in the Normative Aging Study. Am J Clin Nutr March. 1996;63(3):306-14.

21. Blundo C, Marin D, Ricci M. Vitamin B12 deficiency associated with symptoms of frontotemporal dementia. Neurol Sci. 2011 Feb;32(1):101-5. DOI 10.1007/ s10072-010-0419-X.

22. Spence JD. Metabolic vitamin B12 deficiency: a missed opportunity to prevent dementia and stroke. Nutr Res. 2016 Feb;36(2):109-16. DOI 10.1016/j.nutres.2015.10.003.

23. Etogen T, Sander D, Bickel H, Förstl H. Mild cognitive impairment and dementia: the importance of modifiable risk factors. Dtsch ArztebI Int. 2011 Nov;108(44):743-50. DOI 10.3238/arztebl.2011.0743.

24. Ladika DJ, Gurevitz SL. Identifying the most common causes of reversible dementias: a review. JAAPA. 2011 Mar:24(3):28-31, 57.

25. Clarke R, Birks J, Nexo E, Ueland PM, Schneede J, Scott J, et al. Low vitamin B-12 status and risk of cognitive decline in older adults. Am J Clin Nutr. 2007 Nov;86(5):1384-91.

26. Engelboroghs S, Vloeberohs E, Maertens K, Mariën P, Somers N, Symons A, et al. Correlations between cognitive, behavioural and psychological findings and levels of vitamin B12 and folate in patients with dementia. Int J Geriatr Psychiatry. 2004 Apr; 19(4):365-70.

27. van Dyck CH, Lyness JM, Rohrbaugh RM, Siegal AP. Cognitive and psychiatric effects of vitamin B12 replacement in dementia with low serum B12 levels: a nursing home study. Int Psychogeriatr. 2009 Feb;21(1):138-47. DOI 10.1017/S1041610208007904.

28. Seal EC, Metz J, Flicker L, Melny J. A randomized, double-blind, placebo-controlled study of oral vitamin B12 supplementation in older patients with subnormal or borderline serum vitamin B12 concentrations. J Am Geriatr Soc. 2002 Jan;50(1):146-51.

29. Lehmann M, Regland B, Blennow K, Gottfries CG. Vitamin B12-B6-folate treatment improves blood-brain barrier function in patients with hyperhomocysteinaemia and mild cognitive impairment. Dement Geriatr Cogn Disord. 2003;16(3):145-50.

30. van Uffelen JG, Chinapaw MJ, van Mechelen W, Hopman-Rock M. Walking or vitamin B for cognition in older adults with mild cognitive impairment? A randomized controlled trial. Br J Sports Med. 2008 May;42(5):344-51. DOI 10.1136/bjsm.2007.044735.

31. Aisen PS, Schneider LS, Sano M, Diaz-Arrastia R, van Dyck CH, Weiner MF, et al. High-dose B vitamin supplementation and cognitive decline in Alzheimer disease: a randomized controlled trial. JAMA. 2008 Oct;300(15):1774-83. DOI 10.1001/jama.300.15.1774.

32. Brady CB, Gaziano JM, Cxypoliski RA, Guarino PD, Kaufman JS, Warren SR, et al. Homocysteine lowering and cognition in CKD: the Veterans Affairs homocysteine study. Am J Kidney Dis. 2009 Sep;54(3):440-9. DOI 10.1053/j.ajkd.2009.05.013.

33. Martin DC, Francis J, Protetch J, Huff FJ. Time dependency of cognitive recovery with cobalamin replacement: report of a pilot study. J Am Geriatr Soc. 1992 Feb;40(2):168-72.

34. Abyad A. Prevalence of vitamin B12 deficiency among demented patients and cognitive recovery with cobalamin replacement. J Nutr Health Aging. 2002;6(4):254-60.

35. Siswanto O, Smeall K, Watson T, Donnelly-Vanderloo $\mathrm{M}$, O'Connor C, Foley N, et al. Examining the Association between Vitamin B12 Deficiency and Dementia in High-Risk Hospitalized Patients. J Nutr Health Aging. 2015 Dec;19(10):1003-8. DOI 10.1007/s12603015-0531-y.

36. McCracken C. Challenges of long-term nutrition intervention studies on cognition: discordance between observational and intervention studies of vitamin B12 and cognition. Nutr Rev. 2010 Nov;68 SuppI 1:S11-5. DOI 10.1111/j.1753-4887.2010.00325.x.

37. Eastley R, Wilcock GK, Bucks RS. Vitamin B12 deficiency in dementia and cognitive impairment: the 
effects of treatment on neuropsychological function. Int J Geriatr Psychiatry. 2000 Mar;15(3):226-33.

38. Cunha UG. An investigation of dementia among elderly outpatients. Acta Psychiatr Scand. 1990 Sep;82(3):261-3.

39. Cunha UG, Rocha FL, Peixoto JM, Motta MF, Barbosa MT. Vitamin B12 deficiency and dementia. Int Psychogeriatr. 1995 Spring;7(1):85-8.

40. Teunisse S, Bollen AE, van Gool WA, Walstra GJ. Dementia and subnormal levels of vitamin B12: effects of replacement therapy on dementia. J Neurol. 1996 Jul;243(7):522-9.

41. Dangour AD, Allen E, Clarke R, Elbourne D, Fletcher $\mathrm{AE}$, Letley $\mathrm{L}$, et al. Effects of vitamin B-12 supplementation on neurologic and cognitive function in older people: a randomized controlled trial. Am J Clin Nutr. 2015 Sep;102(3):639-47. DOI 10.3945/ajcn.115.110775.

42. Penninx BW, Guralnik JM, Ferrucci L, Fried LP, Allen RH, Stabler SP. Vitamin B(12) deficiency and depression in physically disabled older women: epidemiologic evidence from the Women's Health and Aging Study. Am J Psychiatry. 2000 May;157(5):715-21.

43. Hickie I, Naismith S, Ward PB, Scott E, Mitchell P, Wilhelm K, et al. Vascular risk and low serum B12 predict white matter lesions in patients with major depression. J Affect Disord. 2005 Apr;85(3):327-32.

44. Taylor MJ, Carney SM, Goodwin GM, Geddes JR. Folate for depressive disorders: systematic review and meta-analysis of randomized controlled trials. J Psychopharmacol. 2004 Jun;18(2):251-6.

45. Tiemeier H, van TuijI HR, Hofman A, Meijer J, Kiliaan AJ, Breteler MM. Vitamin B12, folate, and homocysteine in depression: the Rotterdam Study. Am J Psychiatry. 2002 Dec;159(12):2099-101.

46. Kim JM, Stewart R, Kim SW, Shin IS, Yang SJ, Shin HY, et al. Changes in folate, vitamin B12 and homocysteine associated with incident dementia. J Neurol Neurosurg Psychiatry. 2008 Aug;79(8):864-8. DOI 10.1136/ jnnp.2007.131482.

47. Lee SY, Chen SL, Chang YH, Chen SH, Chu CH, Huang SY, et aI. The ALDH2 and DRD2/ANKK1 genes interacted in bipolar II but not bipolar I disorder. Pharmacogenet Genomics. 2010 Aug;20(8):500-6. DOI 10.1097/ FPC.0bo13e32833caa2b.

48. Ng TP, Feng L, Niti M, Kua EH, Yap KB. Folate, vitamin B12, homocysteine, and depressive symptoms in a population sample of older Chinese adults. J Am Geriatr Soc. 2009 May;57(5):871-6.

49. Skarupski KA, Tangney C, Li H, Ouyang B, Evans DA, Morris MC. Longitudinal association of vitamin B-6, folate, and vitamin B-12 with depressive symptoms among older adults over time. Am J Clin Nutr. 2010 Aug;92(2):330-5. DOI 10.3945/ajcn.2010.29413.

50. Levitt AJ, Joffe RT. Folate, B12, and life course of depressive illness. Biol Psychiatry. 1989 Apr;25(7):867-72.

51. Ford AH, Flicker L, Thomas J, Norman P, Jamrozik K, Almeida OP. Vitamins B12, B6, and folic acid for onset of depressive symptoms in older men: results from a 2-year placebo-controlled randomized trial. J Clin Psychiatry. 2008 Aug;69(8):1203-9.

52. Adhikari PM, Chowta MN, Ramapuram JT, Rao SB, Udupa K, Acharya SD. Effect of Vitamin B12 and folic acid supplementation on neuropsychiatric symptoms and immune response in HIV-positive patients. J Neurosci Rural Pract. 2016 Jul-Sep;7(3):362-7. DOI 10.4103/0976-3147.182774.

53. Edney LC, Burns NR, Danthiir $\nabla$. Subjective well-being in older adults: folate and vitamin B12 independently predict positive affect. Br J Nutr. 2015 Oct; 114(8):13218. DOI 10.1017/S0007114515002949.

54. Hintikka J, Tolmunen T, Tanskanen A, Viinamäki H. High vitamin B12 level and good treatment outcome may be associated in major depressive disorder. BMC Psychiatry. 2003 Dec;3:17.

55. Kuo SC, Yeh CB, Yeh YW, Tzeng NS. Schizophrenia-like psychotic episode precipitated by cobalamin deficiency. Gen Hosp Psychiatry. 2009 Nov-Dec;31(6):586-8. DOI 10.1016/j.genhosppsych.2009.02.003.

56. Rajkumar AP, Jebaraj P. Chronic psychosis associated with vitamin B12 deficiency. J Assoc Physicians India. 2008 Feb;56:115-6.

57. Harrington AL, Dixon TM, Ho CH. Vitamin B(12) deficiency as a cause of delirium in a patient with spinal cord injury. Arch Phys Med Rehabil. 2011 Nov;92(11):1917-20. DOI 10.1016/j.apmr.2011.06.003.

58. Nilsson K, Warkentin S, Hultbero B, Fäldt R, Gustafson L. Treatment of cobalamin deficiency in dementia, evaluated clinically and with cerebral blood flow measurements. Aging (Milano). 2000 Jun;12(3): 199-207.

59. Kwok T, Lee J, Lam L, Woo J. Vitamin B(12) supplementation did not improve cognition but reduced delirium in demented patients with vitamin 
B(12) deficiency. Arch Gerontol Geriatr. 2008 MayJun;46(3):273-82.

60. Dos Santos A, Da Silva JA, Almeida J, Corrêa BB, Gago J, Xavier M. Bipolar disorder, homocysteine and white matter hyperintensities. Bipolar Disord. 2008 Sep;10(6):748-9. DOI 10.1111/j.13995618.2008.00595.x.

61. Jacobs LG, Bloom HG, Behrman FZ. Mania and a gait disorder due to cobalamin deficiency. J Am Geriatr Soc. 1990 Apr;38(4):473-4.

62. Goggans FC. A case of mania secondary to vitamin B12 deficiency. Am J Psychiatry. 1984 Feb;141(2):300-1.

63. Issac TG, Soundarya S, Christopher R, Chandra SR. Vitamin B12 deficiency: an important reversible comorbidity in neuropsychiatric manifestations. Indian J Psychol Med. 2015 Jan-Mar;37(1):26-9. DOI 10.4103/0253-7176.150809.

64. Catalano G, Catalano MC, Rosenberg EI, Embi PJ, Embi CS. Catatonia. Another neuropsychiatric presentation of vitamin B12 deficiency? Psychosomatics. 1998 Sep-Oct;39(5):456-60.

65. Zhang X, Hodgson NW, Trivedi MS, Abdolmaleky HM, Fournier M, Cuenod M, et al. Decreased Brain Levels of Vitamin B12 in Aging, Autism and Schizophrenia. PLoS One. 2016 Jan;11(1):e0146797. DOI 10.1371/ journal.pone.0146797.

66. Sharma V, Biswas D. Cobalamin deficiency presenting as obsessive compulsive disorder: case report. Gen Hosp Psychiatry. 2012 Sep-Oct;34(5):578.e7-8. DOI 10.1016/j.genhosppsych.2011.11.006.

67. Lev-Ran S, Le Foll B, McKenzie K, George TP, Rehm J. Bipolar disorder and co-occurring cannabis use disorders: characteristics, co-morbidities and clinical correlates. Psychiatry Res. 2013 Oct;209(3):459-65. DOI 10.1016/j.psychres.2012.12.014.

68. Jauhar S, Blackett A, Srireddy P, McKenna PJ. Pernicious anaemia presenting as catatonia without signs of anaemia or macrocytosis. Br J Psychiatry. 2010 Sep;197(3):244-5. DOI 10.1192/bjp.bp.108.054072.

69. Berry N, Sagar R, Tripathi BM. Catatonia and other psychiatric symptoms with vitamin B12 deficiency. Acta Psychiatr Scand. 2003 Aug; 108(2):156-9.

70. Silverman JJ, Galanter M, Jackson-Triche M, Jacobs DG, Lomax JW 2nd, Riba MB, et al. The American
Psychiatric Association Practice Guidelines for the Psychiatric Evaluation of Adults. Am J Psychiatry. 2015 Aug; 172(8):798-802. DOI 10.1176/appi. ajp.2015.1720501.

71. Robins Wahlin TB, Wahlin A, Winblad B, Bäckman L. The influence of serum vitamin B12 and folate status on cognitive functioning in very old age. Biol Psychol. 2001 Jun;56(3):247-65.

72. Savage DG, Lindenbaum J, Stabler SP, Allen RH. Sensitivity of serum methylmalonic acid and total homocysteine determinations for diagnosing cobalamin and folate deficiencies. Am J Med. 1994 Mar;96(3):239-46.

73. Nurnberger JI Jr, Blehar MC, Kaufmann CA, YorkCooler C, Simpson SG, Harkavy-Friedman J, et al. Diagnostic interview for genetic studies. Rationale, unique features, and training. NIMH Genetics Initiative. Arch Gen Psychiatry. 1994 Nov;51(11):849-59; discussion 863-4.

74. Hølleland G, Schneede J, Ueland PM, Lund PK, Refsum H, Sandberg S. Cobalamin deficiency in general practice. Assessment of the diagnostic utility and cost-benefit analysis of methylmalonic acid determination in relation to current diagnostic strategies. Clin Chem. 1999 Feb;45(2):189-98.

75. Becker M, Axelrod DJ, Chism K, Weinberoer TE, Markov D, Denysenjo L, et al. Hematologic Disorders. In: Fogel BS, Greenberg DB, editors. Psychiatric Care of the Medical Patient. 3th ed. Oxford University Press Inc; 2015. P. 1201.

76. Allen LH. How common is vitamin B-12 deficiency? Am J Clin Nutr. 2009 Feb;89(2):693S-6S. DOI 10.3945/ ajcn.2008.26947A.

77. Vidal-Alaball J, Butler CC, Canninoss-John R, Goringe $\mathrm{A}$, Hood $\mathrm{K}$, McCaddon $\mathrm{A}$, et al. Oral vitamin B12 versus intramuscular vitamin B12 for vitamin B12 deficiency. Cochrane Database Syst Rev. 2005 JuI;(3):CD004655.

78. Mendoza-Bermudez C, de la Espriella-Perdomo $M$. Manifestaciones neuropsiquiátricas del déficit de vitamina B12. Rev Colomb Psiquiatr. 2008;37(1):129-39.

79. Martínez-Marín JD, Henao-Riveros SC, Rey-Tovar MH. Niveles de vitamina B12 en pacientes colombianos con gastritis crónica atrófica. Rev Col Gastroenterol. 2010;25(3):261-4. 